\title{
Le ministère des chantiers
}

\section{Donat Martineau}

Volume 49, 1982

URI : https://id.erudit.org/iderudit/1007091ar

DOI : https://doi.org/10.7202/1007091ar

Aller au sommaire du numéro

Éditeur(s)

Les Éditions Historia Ecclesiæ Catholicæ Canadensis Inc.

ISSN

0318-6172 (imprimé)

1927-7067 (numérique)

Découvrir la revue

Citer cet article

Martineau, D. (1982). Le ministère des chantiers. Sessions d'étude - Société canadienne d'histoire de l'Église catholique, 49, 59-68.

https://doi.org/10.7202/1007091ar

Tous droits réservés @ Les Éditions Historia Ecclesiæ Catholicæ Canadensis Inc., 1982
Ce document est protégé par la loi sur le droit d'auteur. L'utilisation des services d'Érudit (y compris la reproduction) est assujettie à sa politique d'utilisation que vous pouvez consulter en ligne.

https://apropos.erudit.org/fr/usagers/politique-dutilisation/ 


\section{Le ministère des chantiers}

Le ministère des chantiers connut dans l'est du Canada son siècle d'héroïsme et il convient qu'un congrès d'histoire religieuse tenu dans la région dominée durant un demi-siècle par ce ministère y consacre une étude.

\section{ORIGINES}

Ce ministère typiquement canadien-français doit son origine à une inspiration pastorale de $\mathrm{M}^{\mathrm{gr}}$ Bourget en $1841^{1}$. Il visitait la rive nord de la Grande Rivière (Outaouais), section du Bas-Canada. La rive sud ou celle du Haut-Canada avec Bytown (Ottawa) comme centre était sous la juridiction de l'évêque de Kingston, unique évêque du Haut-Canada. Des milliers de Canadiens-français, souvent adolescents, couvraient la rivière Outaouais jusqu'au Témiscamingue et ses affluents. Leurs connaissances religieuses se limitaient au vocabulaire du blasphème et leur culte à celui de Dionysos ou dieu du vin.

Le nouvel évêque de Montréal s'en ouvrit au curé d'Aylmer, Hull n'existant pas encore. L'on constata que la situation était spirituellement pénible. Au printemps le bûcheron devenait "draveur", séjournait des semaines entières à la tête des grands rapides où les cages étaient victimes de l'embouteillage en route pour Québec, vivait dans une oisiveté immorale pour retourner au chantier l'hiver revenu. Les curés du BasCanada redoutaient le retour de ces paroissiens spirituellement gangrenés.

La solution de $\mathbf{M}^{\mathrm{gr}}$ Bourget fut de visiter les bucherons dans leur milieu de travail et de séjourner avec les draveurs dans leur halte prolongée à la tête des rapides. Par ailleurs les missions des Algonquins fondées en 1836 dans le même territoire ne retenaient leurs missionnaires que durant les trois mois d'été. Serait-il possible d'assurer la visite des

${ }^{1}$ Sur la période des débuts (1843-1846), voir Henri MORISSEAU, o.m.i., «Les Oblats dans les chantiers», Revue de l'Université d'Ottawa, 15 (avril-juin 1945), p. 198-216. 
Indiens l'été tandis que leurs missionnaires seraient l'hiver au service des bûcherons et le printemps à celui des draveurs? L'on constata que le clergé séculier était à bout de souffle pour assurer la relève des missions algonquines et que la mission des chantiers requérait au minimum deux missionnaires pour débuter. Le projet fut ajourné.

En 1843, $\mathrm{M}^{\mathrm{gr}}$ Bourget s'en ouvrit à l'évêque de Marseille dont les Oblats venaient d'arriver au Canada et la double oeuvre fut acceptée sans hésitation. En mai $1 \overline{8} \dot{4} 4$, trois Öbiats inauguraient au Canada ie ministère des Indiens : le Père Fisette au Saguenay, le Père Bourassa au Haut du St-Maurice, et le Père Laverlochère au Témiscamingue, en Abitibi et sur la Gatineau. Le 22 janvier suivant, soit en 1845, les Pères Eusèbe Durocher (frère de la Bse Marie-Rose) et Augustin Brunet quittaient Bytown en traîneau pour les chantiers de la Gatineau, randonnée qui devait les conduire au Désert, aujourd'hui Maniwaki. L'oeuvre des chantiers, rêve de $\mathbf{M}^{\mathrm{gr}}$ Bourget, était inaugurée. Signalons en passant que c'était pour assurer cette oeuvre des chantiers que $\mathbf{M}^{\mathrm{gr}}$ Bourget avait fortement recommandé l'établissement des Oblats à Ottawa auprès de l'évêque de Kingston. Bytown était alors le centre des chantiers de l'Outaouais.

\section{DESCRIPTION DU MINISTÈRE}

Sur la tombe encore ouverte du grand missionnaire de chantiers Louis Reboul, o.m.i., son émule Jean-Marie Nédélec, o.m.i. faisait la description de ce ministère :

En arrivant dans un chantier vous vous trouvez en face d'abord de gens de toutes nations; Canadiens français, Irlandais, Écossais, etc. etc., des personnes de toute religion, protestants de toute dénomination sans compter les catholiques, des personnes de tout âge, de tout caractère. Pour ne pas blesser, froisser personne, pour plaire à tout le monde autant que cela peut [se faire], cela demande un grand tact, une grande expérience, un grand savoir-faire. Sans cette connaissance des hommes et des choses, on ne ferait pas beaucoup de bien, on pourrait faire beaucoup de mal : point à observer. Voilà pourquoi les missions de chantier demandent des missionnaires pleins de tact, pleins d'esprit d'observation pour deviner ce qu'il faut faire suivant les temps, les personnes, les lieux, les circonstances et tout cela sous peine de rendre ces missions inutiles mais encore désagréables. En un mot le ministère des chantiers demande un ensemble de qualités particulières : une grande patience pour tout endurer, une grande connaissance du coeur humain pour savoir quelle fibre toucher, une 
grande gaieté pour tout enchanter et amuser, surtout un coup d'oeil exercé pour savoir que dire, que faire : et par dessus tout une grande santé pour endurer le chaud et le froid, la fatigue et les autres privations inséparables de la vie des missionnaires, surtout dans les bois. ${ }^{2}$

Qu'il nous soit permis d'assister au départ de Bytown le 22 janvier 1845 des deux initiateurs de ce ministère. Le traîneau conduit par un charretier se présente à la résidence. Une valise pour le linge est placée à l'arrière et sert de siège. Le colis contenant les livres de chants et de prières sert de dossier. Au centre l'on place un coffre ou chapelle portative contenant la pierre d'autel, les vêtements réversibles aux cinq couleurs, les burettes, missel, nappes d'autels, etc. Voici les missionnaires en route. À ciel ouvert ils devront affronter les tempêtes et le froid, se relever allègrement si la voiture culbute dans les soubresauts des bancs de neige, atteindre le premier chantier vers la fin de l'après-midi, sourire à tous malgré la fatigue souvent doublée d'une migraine, partager les lourds mets monotones des bûcherons, diriger la conversation de la soirée, animer les chants, adapter au groupe l'unique sermon qui devra être vibrant et soulever les consciences, entendre les longues confessions, enfin coucher vers minuit sur la dure, supporter le froid ou un excès de chaleur, se lever vers quatre heures, monter la chapelle, célébrer la messe et donner les dernières recommandations, saluer chacun des bûcherons, retournant joyeux au bois, recharger le traîneau et repartir pour un autre chantier où il faudra arriver avant le coucher hâtif du soleil. La visite portera de 40 à 60 chantiers, et sera exécutée en autant de jours.

Ce n'est pas sans hésitation que je présente trois héros légendaires de ce ministère à des historiens chevronnés et assez perspicaces pour discerner entre l'authenticité et la panégyrisme enthousiaste. Cependant s'il est possible que la légende ait multiplié le nombre des Anglais culbutés en bas du pont des Chaudières par Jos. Monferrant et accentué le trou fait par le talon du bûcheron dans le plafond de l'auberge outaouaise, l'on ne peut nier son existence et sa force musculaire. Dans la présentation de nos trois héros légendaires, je dois accepter d'être accusé de naïveté en acceptant comme fondamentalement vrais des faits invraisemblables mais corroborés par des témoins que l'historien le plus critique doit accepter comme dignes de foi.

${ }^{2}$ Codex historicus de Mattawa, p. 26-28, cité par G. CARRIÈRE, o.m.i., Le Père Louis-Étienne Reboul..., p. 34-35. 
Né en Bretagne en 1827 et ordonné prêtre oblat en 1852, Louis Reboul arrivait à Bytown en 1853. D'abord desservant de South Gloucester et des postes environnants, il se rendait à Maniwaki en 1856. C'était le centre des chantiers de la Gatineau et de la Lièvre, des postes de fourrures et des établissements des colons échelonnés le long de la Gatineau et de la Désert. Les missionnaires de Maniwaki desservaient même le Haut du St-Maurice. Gräce à sa force muscuiaire, Louis Reboui entrait dans la légende dès l'âge de 29 ans. À sa mort on écrivait de lui :

Le Père Reboul commença dès lors à faire preuve d'une indomptable énergie. Il se faisait tout à tous, bûcheron, défricheur, cultivateur et ne se reposait de ces occupations nouvelles que par la visite des malades et la desserte des différents postes qui dépendaient de la mission. La plupart se trouvaient éloignés de 20,30, 40 milles de sa résidence; pas de chemin pour s'y rendre, il fallait parcourir la distance à pied ou en canot d'écorce. Le Père Reboul prenait l'aviron, et parcourait les lacs, les rivières, sautait les rapides, exécutait lui-même ses portages, étonnant les sauvages eux-mêmes par son adresse et son énergie. ${ }^{4}$

En 1858, il est nommé chef des missionnaires des chantiers avec résidence à la cathédrale d'Ottawa. Son apostolat couvrait trois facettes : la visite des chantiers, celle des draveurs et l'apostolat auprès des jeunes qui inondaient la ville.

La visite des chantiers était annuelle. En 1863, il en visita 55 en 60 jours, 60 en 1865 et 80 en 1870 . Il se devait de toujours se présenter en excellente forme physique puisqu'à son arrivée il était attendu pour la démonstration de la tire du poignet de laquelle il sortit toujours vainqueur. Les applaudissements ouvraient les consciences.

À l'arrivée des cages des draveurs ou des trains flottants, il passait de l'une à l'autre, les escaladait, faisait réfléchir les draveurs sur les graves dangers de leur profession (en une seule saison 200 avaient som-

${ }^{3}$ Gaston CARRIÈRE, o.m.i., Le Père Louis-Étienne Reboul, o.m.i., (1827-1877), organisateur de la vie religieuse de Hull et apôtre des chantiers, Ottawa, Éditions de l'Université d'Ottawa, 1959, 157 p.

${ }^{4}$ Notices nécrologiques, vol. 3 , p. 354 , cité par G. CARRIÈRE, Le Père LouisÉtienne Reboul..., p. 23. 
bré dans les flots de l'Outaouais et de ses affluents) ou les réunissait dans leur chapelle spéciale construite à Hull (ce fut la première de la ville) durant leur long arrêt.

Son ministère couvrait les milliers de jeunes qui se tenaient dans les auberges et hôtels d'Ottawa en attendant leur embauchage. Périodiquement il leur prêchait des retraites au sous-sol de la cathédrale. Il passait de chambre en chambre, d'hôtel en hôtel et amenait chacun entendre la parole de Dieu. C'est dans ce genre de ministère qu'il fut auréolé par la légende. Le mélange des catholiques et des protestants, des Canadiens, des Irlandais et des Anglais était l'occasion de rixes, de batailles et même d'émeutes. À ce sujet les rôles du pasteur et des forces de l'ordre étaient renversés : ce n'est pas le Père Auboul (ainsi était-il appelé) qui faisait appel aux policiers mais ceux-ci, débordés et impuissants, faisaient intervenir l'hypnotiseur des foules. Je laisse tomber la légende et me limite à deux faits officiels. Son Supérieur, le Père Grenier, écrit au Supérieur Général des Oblats et rapporte les événements suivants. ${ }^{5}$

Le 12 juillet 1865 c'était la grande fête des Orangistes. Après la procession et le banquet de 6,000 convives, des Canadiens et des Irlandais entrent en scène. On s'arme de pierre, de bâtons et on demande des fusils. Reboul est demandé sur les lieux. Le président, jouant le rôle de Guillaume le Conquérant, le supplie de ne pas quitter les lieux. Les catholiques attendaient son départ pour se précipiter sur les Orangistes. Il réussit à les contenir. Il devient alors l'idole tant des protestants que des catholiques. Un duel fut un jour décidé entre deux jeunes. Une petite île entre les deux villes est choisie comme lieu idéal. Deux cents témoins avides de sensations sont sur les lieux. Alerté le Père Reboul s'y rend en canot. À sa vue, le groupe se disperse.

Les draveurs et les bûcherons devaient manifester leur reconnaissance à leur héros d'une manière tangible. Le 7 septembre 1866, ils faisaient ériger au sommet de la cathédrale d'Ottawa entre les deux tours une statue de Marie tenant dans ses bras l'Enfant Jésus tournée vers la Grande Rivière pour solliciter sa protection dans leur dangereux travail.

${ }^{5}$ Le texte de la lettre (15 aout 1865) est cité par G. CARRIÈRE, Le Père Louis-Etienne Reboul..., p. 74-77. 
Afin de consolider l'oeuvre des chantiers, il fut jugé opportun pour les Oblats de s'établir à Hull. Le décret d'établissement fut signé le 14 novembre 1870 et le Père Reboul se mit à l'oeuvre. Entre ses visites des chantiers et des draveurs, son apostolat auprès des jeunes, il organisa si bien la future ville que sa biographie est intitulée "Louis Reboul, o.m.i. organisateur de la vie religieuse à Hull » .

Son legs à Ottawa fut l'oeuvre des Soeurs du Bon Pasteur pour la protection des jeunes filles exposées aux désordres inhérents à la présence des milliers de jeunes étrangers. Il fut l'ange protecteur de l'institution. Le 6 janvier 1877, il fit comme à l'accoutumée la visite hebdomadaire de tout le personnel. C'était sa dernière visite.

En effet, sauf en l'année 1874 au cours de laquelle il dirigeait les travaux de la construction de l'église de Hull, il n'avait jamais omis la visite des chantiers dont il était responsable. Le 11 janvier il partait indisposé pour sa 25ème visite. Il visita le long de la Rivière Outaouais 45 chantiers, souffrant physiquement, se traînant de l'un à l'autre sans cependant omettre la prédication et l'audition des confessions. Finalement dans le secteur de Mattawa il dut célébrer une dernière messe. Il se laissa conduire agonisant à la mission des Oblats de Mattawa, distance de 35 milles. Il reçut l'Extrême-Onction et communia en viatique. Il décédait le 2 mars et fut inhumé à Hull le 7.

\section{JEAN-MARIE NÉDÉLEC, O.M.I., $1834-1896^{6}$}

Né le 8 mai 1834 en Bretagne, J.M. Nédélec fut ordonné prêtre en 1859. Entré chez les Oblats en 1861 il arriva au Canada en mai 1863. D'abord missionnaire sur la côte Nord, il passe à Maniwaki en 1867 où il fait la visite des chantiers en compagnie du P. Reboul. Il arrive au Témiscamingue en 1868 pour prendre la responsabilité des Indiens du lac Abitibi et de la Baie James.

Cependant selon les prévisions de $\mathrm{M}^{\mathrm{gr}}$ Bourget, le missionnaire des Indiens était en mesure d'accepter l'hiver l'oeuvre des chantiers. Cette formule avait un double motif : elle permettait de répondre à la pénurie de personnel pour des oeuvres à demi-temps et elle assurait aux missionnaires des Indiens un revenu appréciable. Les missionnaires des Indiens

${ }^{6}$ Gaston CARRIÈRE, o.m.i., Le voyageur du Bon Dieu, le Père Jean-Marie Nédélec, o.m.i. (1834-1896), Montréal, Ed. Rayonnement, 1961, 162 p. 
dans notre région ont donc eu tendance à résider dans un centre de chantiers. Ainsi la mission Saint-Claude au Témiscamingue, située face au Fort Témiscamingue, était le centre à la fois des missions indiennes et des chantiers. Quant à lui, le Père Nédélec, responsable des missions de la Baie James, établit dès 1869 sa résidence à Mattawa, centre des chantiers de la section ouest du futur diocèse de Pembroke. De même que son émule le P. Reboul fut le fondateur de Hull, le P. Nédélec était appelé à fonder Mattawa et y être le premier prêtre résident.

Il loge tout d'abord dans une maisonnette prêtée par Noé Timmins, Canadien français dont le fils devait donner le nom à la future ville minière de Timmins. De 1869 à 1892 le P. Nédélec fut l'animateur de Mattawa et de ses environs. Il exerça son ministère à Eau Claire, aux Joachims, à Golden Lake, à Maynooth, McKey's Station, etc. Tout en visitant les chantiers de la région, il organise les résidents dont le nombre augmente graduellement. Il voit à la construction de leur chapelle. S'il revient à son centre, c'est pour se muter en professeur bénévole pour ses élèves français, irlandais et indiens avant l'arrivée des institutrices et des Soeurs Grises de la Croix.

Non satisfait de ce surmenage intempestif, il accepte le plus pénible des ministères, celui de constructeurs de chemin de fer de Pembroke à North Bay de 1879 à 1883. En 1880, 2000 cheminots catholiques travaillaient dans le secteur ferroviaire de Mattawa, sans compter les familles qui se fixaient à proximité des futures stations : Bisset's Creek, Rockliffe, les Deux-Rivières, Eau-Claire, etc. En octobre 1883 il dépassait inconsciemment sa juridiction. Les cheminots avaient atteint le site de la future ville de North Bay. Le Père ne réalisant pas qu'il avait franchi le diocèse de Peterborough dont devait se détacher par la suite Sault-Ste-Marie, célébrait la première messe de la ville : il chantait la messe en pleine air sur les bords du lac Nipissing devant 800 hommes.

En 1892, il remet les missions de la Baie James qu'il avait visitées 22 fois en partance de Mattawa entre les mains du premier missionnaire résident à la Baie James, le Père Frs-X. Fafard, o.m.i.. Il laisse également Mattawa pour desservir la réserve des Indiens du Témiscamingue fixée à la Tête du Lac ou Notre-Dame du Nord. De passage à Mattawa en 1896, il y décède le 23 février à 61 ans. Il y fut inhumé. Coïncidence touchante, il décédait dans la maison où 19 ans auparavant il avait présenté le saint viatique à son émule Louis Reboul, o.m.i.. 


\section{JEAN-PIERRE GUÉGUEN, O.M.I., 1838-1909 ${ }^{7}$}

Si le Père Reboul eut pour théâtre Hull et ses environs, le Père Nédélec Mattawa, le Père Guéguen eut en partage les chantiers du Témiscamingue et de la région de Maniwaki sur la Gatineau tout en évangélisant l'été les Indiens. Sa biographie est ainsi préfacée : «Gravement malade de naissance, il a mérité le titre de voltigeur, cavalier romain habile à faire une «volte-face» rapide sur son coursier. Trente-trois fois notre voltigeur intrépide parcourut en canot le circuit de la vallée de l'Outaouais supérieur et de la vallée de la Gatineau, circuit que parcourt le touriste actuel de Mattawa à Maniwaki». ${ }^{8}$ Ses courses annuelles s'étendaient jusqu'au haut du St-Maurice atteignant même le versant de la Baie James.

Afin de saisir le pourquoi géographique de ce circuit du Père Guéguen nous devons rappeler que le Parc LaVérendrye est un grand plateau où les sources des grandes artères fluviales québécoises se donnent la main. Du Grand Lac Victoria, source de la rivière Ottawa, l'on passe par un simple portage aux sources de la Gatineau. Celles-ci voisinent les sources du St-Maurice, voisines à leur tour d'un affluent du lac StJean et du lac Waswanipi qui se déverse à la Baie James. Ce plateau central, point de jonction des missions indiennes québécoises était donc le carrefour des missionnaires et explique comment jusqu'en $1938 \mathrm{La}$ Tuque était dans le diocèse de Haileybury.

Né en Bretagne le 18 septembre 1838, Jean-Pierre Guéguen entra chez les Oblats en 1860. Il arrive au Témiscamingue en 1865. Un à un il visita les 4,000 bûcherons échelonnés en petits groupes autour du lac et des affluents de 1865 à 1886 , année où il se rendit à Maniwaki y remplir le même ministère.

Le charisme du Père Guéguen fut d'être porteur d'une grave maladie incurable inconciliable avec son double ministère. Il ne fut pas bénéficiaire d'une guérison miraculeuse mais victime d'une maladie miraculeuse. Trente-trois fois il fit l'été le voyage du Témiscamingue au Haut du St-Maurice, voyage estimé par $\mathrm{M}^{\mathrm{gr}}$ Lorrain à 1700 milles, soit en tout deux fois le tour du monde. Voyons en quel état physique :

${ }^{7}$ Gaston CARRIÈRE, o.m.i., Le Père Jean-Pierre Guéguen, o.m.i., 1838-1909, un grand voltigeur (Mattawa, Kipawa, Tête du Lac, Weymontaching, Maniwaki), Guérin, Ed. de la Société historique, Rivière des Quinze, 1978, 190 p.

8 G. CARRIÈRE, Le Père Jean-Pierre Guéguen..., p. 3. 
Le Rév. Père Guéguen est toujours entre la vie et la mort [le Père devait survivre 37 ans à cette lettre]. À la maison, il garde presque toujours le lit. Dès qu'il s'agit d'aller en mission, il est debout. Il est incompréhensible. Tout chétif et maladif, il a une énergie qui triomphe de tout. Personne mieux que lui domine les sauvages quant au spirituel. ${ }^{9}$

À 67 ans, il écrivait de lui-même à son provincial :

J'ai toujours été faible depuis que je suis au monde, mais j'ai toujours mis ma confiance en Dieu. Aussi quand je me suis trouvé incapable de remuer, comme en 1870 et 1872 que j'ai été 5 jours cloué sur un plancher de la mission de Kikendatch sur le StMaurice, j'ai espéré en lui et la sixième journée je continuais mon chemin comme si rien était arrivé! Mais quand tout va à merveille, je m'attends à quelque surprise comme celle qui m'est arrivée à Mattawa où les sauvages m'ont cru mort à l'Église pendant quelque temps. Dieu merci, je n'ai rien ressenti depuis ce temps-là. ${ }^{10}$

Le compagnon de son dernier voyage de 1500 milles fait la description d'un portage d'un lac à un autre ou d'un chantier au suivant :

Lorsque je l'accompagnais (c'était son dernier voyage), c'était pitié de le voir et toujours, il était de bonne humeur. On l'attendait à chaque portage, cependant les Indiens faisaient 3 ou 4 voyages par portage. Une fois, on l'attendit près d'une heure, c'était un portage long et fangeux; il faisait une chaleur étouffante et les mouches le poursuivaient; il arriva au canot, épuisé (on dut le porter), on l'aida à embarquer et il se coucha dans le canot en disant : "Poor me», c'était sa plainte. On m'a dit que quelques fois, il ne pouvait marcher. Alors les Indiens, qui le vénéraient, le portaient sur un hamac fait de deux perches et d'une couverture de laine. ${ }^{11}$

Sa résistance l'a soutenu jusqu'à sa mort survenue le 22 octobre 1909 à 71 ans. Le 31 mai précédent, on écrit de lui dans le Codex historicus de Maniwaki : "Le Dr Mulligan trouve que le Père Guéguen toujours mieux. Il s'étonne de la vitalité de ce vieillard. Il n'explique

9 Père F. Vandenberghe, provincial, au Père J. Fabre, supérieur général, 17 septembre 1872, cité par G. CARRIÈRE, Le Père Jean-Pierre Guéguen..., p. 10-11.

${ }^{10}$ Ibidem, p. 173.

${ }^{11}$ Ibidem, p. 186. 
son rétablissement que comme un effet de la force de sa volonté. Au moins trois fois depuis deux ans, le Père Guéguen semblait ne pouvoir se rétablir, et chaque fois qu'il a repris le dessus, le $\mathrm{Dr}$ et les autres ont été étonnés». Et le 3 juin, on note encore : «Le Père Guéguen a fait des progrès étonnants. On dirait qu'il va se rétablir. Comme de raison, il n'est pas bâti comme un autre. Ce n'est pas la première fois qu'il déroute les médecins et qu'il crache sur leurs sentences de mort». ${ }^{12}$

Terminons en rappelant que le ministère des chantiers n'existe plus. La mécanisation, l'ouverture des chemins et l'envahissement des forêts par la civilisation permettent aux bûcherons le retour hebdomadaire à leurs foyers. Ayant débuté en 1845, il s'est prolongé durant 125 ans pour s'éteindre graduellement et disparaitre.

Donat Martineau, o.m.i., Société du Patrimoine de

l'Abitibi-Témiscamingue, Guérin (Cté Témiscamingue).

12 Ibidem, p. 20. 